\title{
Supernova Neutrino Detection in Borexino
}

\author{
L. Cadonati ${ }^{1}$, F.P. Calaprice, M.C. Chen ${ }^{2}$ \\ Department of Physics, Jadwin Hall, Princeton University, Princeton, NJ 08544
}

\begin{abstract}
We calculated the expected neutrino signal in Borexino from a typical Type II supernova at a distance of $10 \mathrm{kpc}$. A burst of around 110 events would appear in Borexino within a time interval of about $10 \mathrm{~s}$. Most of these events would come from the reaction channel $\bar{\nu}_{e}+p \rightarrow e^{+}+n$, while about 30 events would be induced by the interaction of the supernova neutrino flux on ${ }^{12} \mathrm{C}$ in the liquid scintillator. Borexino can clearly distinguish between the neutral-current excitations ${ }^{12} \mathrm{C}\left(\nu, \nu^{\prime}\right){ }^{12} \mathrm{C}^{*}(15.11 \mathrm{MeV})$ and the charged-current reactions ${ }^{12} \mathrm{C}\left(\nu_{e}, e^{-}\right){ }^{12} \mathrm{~N}$ and ${ }^{12} \mathrm{C}\left(\bar{\nu}_{e}, e^{+}\right){ }^{12} \mathrm{~B}$, via their distinctive event signatures. The ratio of the chargedcurrent to neutral-current neutrino event rates and their time profiles with respect to each other can provide a handle on supernova and non-standard neutrino physics (mass and flavor oscillations).
\end{abstract}

Key words: Neutrino detector, Supernovae, Neutrino mass PACS: 14.60.Pq, 25.30.Pt, 95.55.Vj, 97.60.Bw

1 Corresponding author: Department of Physics, Jadwin Hall, Princeton University, Princeton, NJ 08544, USA; telephone 1-609-258-1122; fax 1-609-258-1356 email cadonati@princeton.edu

2 Present address: Department of Physics, Stirling Hall, Queen's University, Kingston, Ontario, Canada.

Preprint submitted to Astroparticle Physics 9 November 2018 


\section{Introduction}

The last supernova in our galaxy that was visible on Earth was observed in 1604. Back then, astronomers marveled at "Kepler's Star" in Ophiuchus and studied the light curve from the colossal explosion. The next Galactic supernova will differ from previous ones in human history. Not only will astronomical observations be made across the electromagnetic spectrum; it is likely that several neutrino detectors will detect the burst of neutrinos emerging from the stellar gravitational collapse. Indeed, it may turn out that the next Galactic supernova will not be seen optically, due to obscuration (as was probably the case for the radio source supernova remnant Cassiopeia A), and that supernova neutrino detection will signal the astrophysical event, triggering its search in electromagnetic bands.

The field of extrasolar neutrino astrophysics was born when neutrinos from SN1987A in the Large Magellanic Cloud were detected by the IMB [1] and Kamiokande [2] neutrino detectors. These pioneering observations contributed significantly to our understanding of the mechanisms involved in a supernova explosion, as well as providing interesting limits on neutrino properties. The next Galactic supernova will prove even more valuable owing to the abundance of neutrino events produced by the closer source and the variety of reactions that will be available to study these neutrinos.

SuperKamiokande [3] and SNO [4] will be the largest supernova neutrino detectors in the foreseeable future. SuperKamiokande contains about 50,000 tons of water, providing proton targets for the inverse $\beta$ decay reaction. The SNO detector is filled with a kiloton of heavy water that offers deuterons for neutrino interactions. Detectors employing liquid scintillator that will be capable of detecting supernova neutrinos include Baksan [5], LVD [6], MACRO [7], Borexino [8], and KamLAND [9]. In a liquid scintillator (typical composition $\mathrm{CH}_{2}$ ), the antineutrino-proton reactions will still constitute the majority of the detected supernova neutrino events. Nevertheless, the abundance of carbon in an organic liquid scintillator provides an additional, interesting target for neutrino interactions [10].

Neutrino reactions on the ${ }^{12} \mathrm{C}$ nucleus $\left(\mathrm{J}^{\pi}=0^{+}, \mathrm{T}=0\right)$ include the superallowed transitions to the $\mathrm{A}=12$ triad $\left(\mathrm{J}^{\pi}=1^{+}, \mathrm{T}=1\right)$ of ${ }^{12} \mathrm{~B}$ (ground state), ${ }^{12} \mathrm{C}^{*}(15.1 \mathrm{MeV})$ and ${ }^{12} \mathrm{~N}$ (ground state). The cross sections for these reactions are reasonably large, for neutrinos above the reaction thresholds of 14.4, 15.1, and $17.3 \mathrm{MeV}$, respectively. This system is in many ways ideal for investigating neutrino physics since it offers charged-current and neutral-current neutrino reactions, allowing one to probe for potential neutrino flavor oscillations. In addition, each of the above reactions has a distinguishing detection signature. In the case of the charged-current reactions, the signature is the electron (or 
positron) emitted in the prompt reaction, followed tens of milliseconds later by the $\beta^{+}$decay (or $\beta^{-}$decay) back to ${ }^{12} \mathrm{C}$ - a delayed coincidence that enables identification of these specific interactions from background. For the neutral-current excitation of ${ }^{12} \mathrm{C}$, the $15.1 \mathrm{MeV}$ gamma following de-excitation is the signature.

In a Type II supernova, neutrinos are emitted with a thermal spectrum. The average neutrino energy depends upon the species of neutrino, since decoupling in the neutrinosphere occurs at different temperatures for the different species. Typical values for the average neutrino energies are [11]: $\langle E\rangle=11 \mathrm{MeV}$ for $\nu_{e},\langle E\rangle=16 \mathrm{MeV}$ for $\bar{\nu}_{e}$, and $\langle E\rangle=25 \mathrm{MeV}$ for $\nu_{\mu}, \nu_{\tau}, \bar{\nu}_{\mu}$, and $\bar{\nu}_{\tau}$. This energy hierarchy enhances the sensitivity of the neutrino-carbon reactions to non-standard neutrino physics. The $\nu_{\mu}$ 's and $\nu_{\tau}$ 's are more energetic than electron neutrinos. They dominate the neutral-current reaction ${ }^{12} \mathrm{C}\left(\nu, \nu^{\prime}\right){ }^{12} \mathrm{C}(15.11 \mathrm{MeV})$ with an estimated contribution of around $90 \%$. This allows the ${ }^{12} \mathrm{C} 15.1 \mathrm{MeV}$ events to be closely identified with the "heavy flavor" species. Additionally, since the average energy of the $\nu_{e}$ or $\bar{\nu}_{e}$ from the supernova is at or below their charged-current reaction thresholds, the total number of these events will be small. This provides a pseudo-appearance opportunity for observing neutrino flavor oscillations.

In order to exploit these aspects for exploring non-standard neutrino physics, a liquid scintillator supernova neutrino detector needs to be able to cleanly detect the $15.1 \mathrm{MeV} \gamma$ ray from the neutral-current reaction. This implies that the detector requires a large volume to contain this energetic $\gamma$ ray. Additionally, good energy resolution is necessary for resolution of the peak produced by these events in the energy spectrum. The LVD and MACRO experiments are existing scintillator detectors, but their $15.1 \mathrm{MeV} \gamma$ ray capabilities are somewhat limited since they are both segmented detectors. Borexino and KamLAND will have excellent efficiency for detecting and resolving the $15.1 \mathrm{MeV}$ signal, as they will both feature large, homogeneous volumes of liquid scintillator.

In this paper, we present calculations of the neutrino event rates in Borexino for a hypothetical Type II supernova at a distance of $10 \mathrm{kpc}$, with a typical binding energy release of $3 \times 10^{53} \mathrm{ergs}$. We illustrate the neutral-current capability of Borexino, via detection of the $15.1 \mathrm{MeV} \gamma$ ray. Finally, we describe the capabilities of Borexino for exploring supernova neutrino oscillations and setting stringent neutrino mass limits, based upon the delayed arrival of a heavy $\nu_{\tau}$. Despite being a much smaller detector, the neutrino mass limits to which Borexino is sensitive are comparable to what might be achieved by Super-Kamiokande and SNO, in the event of a Galactic supernova. 


\section{Supernova Neutrino Signatures in Borexino}

The Borexino experiment [8] is under construction at the Laboratori Nazionali del Gran Sasso underground facility, in Italy. Its main physics goal is the detection of the $0.86 \mathrm{MeV} \nu_{e}$ from electron-capture decay of ${ }^{7} \mathrm{Be}$ in the Sun. The detector has a central volume containing 300 tons of liquid scintillator (pseudocumene plus fluor), contained in a spherical nylon bag and viewed by 2,200 photomultiplier tubes.

Supernova neutrinos will interact in the liquid scintillator via electron scattering, inverse $\beta$ decay of the proton and reactions on ${ }^{12} \mathrm{C}$. Constructed as a low-background solar neutrino detector, Borexino will be able to cleanly detect all of these supernova neutrino interactions, with the exception that the handful of electron scattering events have no clear distinguishing signature.

We start by considering a stellar gravitational collapse releasing $\varepsilon_{B} \approx 3 \times$ $10^{53}$ ergs binding energy [12]. $99 \%$ of this binding energy comes off in the form of neutrinos, most of which originate from $\nu \bar{\nu}$ pair production. Only a small fraction (a few percent) of the neutrinos are produced in other processes, such as the neutronization during core collapse.

At core collapse temperatures and densities exceeding $10^{11} \mathrm{~g} / \mathrm{cm}^{3}$, matter is not transparent to neutrinos. Scattering interactions thermalize the neutrinos though their mean free path remains large; in effect, neutrinos become the energy transport agents in the collapsed stellar core. They emerge from the cooling core or "neutrinosphere" after they decouple. The temperature at the time of decoupling determines the energy distribution of the emitted neutrinos. Neutrinos of $\mu$ and $\tau$ flavor decouple at higher temperature, since they interact in ordinary matter only via the neutral-current weak interaction, whereas charged-current scattering can occur for $\nu_{e}$ and $\bar{\nu}_{e}$. Moreover, the neutrino decoupling takes place in neutron rich matter, which is less transparent to $\nu_{e}$ than $\bar{\nu}_{e}$. The temperature hierarchy is, then: $\mathrm{T}_{\nu_{e}}<\mathrm{T}_{\bar{\nu}_{e}}<\mathrm{T}_{\nu_{x}}$, where $\nu_{x}$ denotes $\nu_{\mu, \tau}$ and $\bar{\nu}_{\mu, \tau}$. Each spectrum can be considered as a Fermi-Dirac distribution with zero chemical potential [11]:

$$
\frac{\mathrm{dN}}{\mathrm{dE}_{\nu}}=\frac{0.5546}{\mathrm{~T}^{3}} \frac{\mathrm{E}_{\nu}^{2}}{1+\mathrm{e}^{\mathrm{E}_{\nu} / \mathrm{T}}} \mathrm{N}_{0},
$$

with the following parameters:

$$
\begin{array}{rll}
\nu_{e} & \mathrm{~T}=3.5 \mathrm{MeV} & \left\langle\mathrm{E}_{\nu}\right\rangle=11 \mathrm{MeV} \\
\bar{\nu}_{e} & \mathrm{~T}=5 \mathrm{MeV} & \left\langle\mathrm{E}_{\nu}\right\rangle=16 \mathrm{MeV} \\
\nu_{\mu, \tau} \bar{\nu}_{\mu, \tau} & \mathrm{T}=8 \mathrm{MeV} & \left\langle\mathrm{E}_{\nu}\right\rangle=25 \mathrm{MeV} .
\end{array}
$$


Table 1

Supernova neutrino reactions in Borexino, their energy thresholds and the number of targets in the sensitive volume (300 tons $\left.\mathrm{C}_{9} \mathrm{H}_{12}\right)$.

\begin{tabular}{lcc}
\hline Reaction & $\mathrm{E}_{\text {thres }}$ & Number of targets in 300 tons \\
\hline$\nu+e^{-} \rightarrow \nu+e^{-}$ & 0 & $9.94 \times 10^{31}$ \\
$\bar{\nu}_{e}+p \rightarrow e^{+}+n$ & $1.80 \mathrm{MeV}$ & $1.81 \times 10^{31}$ \\
${ }^{12} \mathrm{C}\left(\nu_{e}, e^{-}\right)^{12} \mathrm{~N}$ & $17.3 \mathrm{MeV}$ & $1.36 \times 10^{31}$ \\
${ }^{12} \mathrm{C}\left(\bar{\nu}_{e}, e^{+}\right)^{12} \mathrm{~B}$ & $14.4 \mathrm{MeV}$ & $1.36 \times 10^{31}$ \\
${ }^{12} \mathrm{C}\left(\nu, \nu^{\prime}\right)^{12} \mathrm{C}^{*}$ & $15.1 \mathrm{MeV}$ & $1.36 \times 10^{31}$ \\
\hline
\end{tabular}

Based upon equipartition, the prediction is that all of the neutrino species are produced in the cooling core with the same luminosity [12]. This implies that the number of $\nu_{e}$ 's will be greater than $\nu_{\mu}$ and $\nu_{\tau}$, since their average energy is lower.

The expected event rates for each of the reactions in Borexino were calculated by integrating the cross sections as functions of energy over the Fermi-Dirac spectra for the relevant neutrino species. The total number of neutrinos of each type was estimated by dividing the binding energy equally between the six $\nu$ or $\bar{\nu}$ species, and then dividing by the average energy $\langle\mathrm{E}\rangle$ of each species. We consider 300 tons of pseudocumene $\left(\mathrm{C}_{9} \mathrm{H}_{12}\right)$ as the target for neutrinos in Borexino and list the reactions, thresholds, and number of targets in Table 1.

\section{$2.1 \nu-e^{-}$scattering}

Neutrino-electron scattering produces recoil electrons with energy from zero up to the kinematic maximum. In Borexino the recoil electron detection threshold will be $0.25 \mathrm{MeV}$, small compared to supernova neutrino energies. In our rate calculation we approximate by integrating over all electron recoil energies. The standard electroweak cross section, with $\mathrm{E}_{\nu} \gg \mathrm{m}_{e}$, is:

$$
\sigma=\frac{2 \mathrm{G}_{\mathrm{F}}^{2} \mathrm{~m}_{e} \mathrm{E}_{\nu}}{\pi}\left[\mathrm{c}_{\mathrm{L}}^{2}+\frac{1}{3} \mathrm{c}_{\mathrm{R}}^{2}\right]
$$

where the coupling constants depend on the neutrino species considered. The total cross sections for $\nu-e^{-}$scattering are linearly proportional to the neutrino energy, and appear with appropriate parameters as:

$$
\begin{aligned}
& \sigma\left(\nu_{e} e \rightarrow \nu_{e} e\right)=9.20 \times 10^{-45} \mathrm{E}_{\nu[\mathrm{MeV}]} \mathrm{cm}^{2} \\
& \sigma\left(\bar{\nu}_{e} e \rightarrow \bar{\nu}_{e} e\right)=3.83 \times 10^{-45} \mathrm{E}_{\nu[\mathrm{MeV}]} \mathrm{cm}^{2}
\end{aligned}
$$




$$
\begin{aligned}
& \sigma\left(\nu_{\mu, \tau} e \rightarrow \nu_{\mu, \tau} e\right)=1.57 \times 10^{-45} \mathrm{E}_{\nu[\mathrm{MeV}]} \mathrm{cm}^{2} \\
& \sigma\left(\bar{\nu}_{\mu, \tau} e \rightarrow \bar{\nu}_{\mu, \tau} e\right)=1.29 \times 10^{-45} \mathrm{E}_{\nu[\mathrm{MeV}]} \mathrm{cm}^{2}
\end{aligned}
$$

For all of the neutrino species from a typical supernova at $10 \mathrm{kpc}$, the calculated event rate in Borexino is about 5 events from neutrino-electron scattering.

\section{2 $\bar{\nu}_{e}+p$ reaction}

The large cross section, low threshold, and abundance of target protons makes this the dominant channel for detection of supernova neutrinos. The inverse $\beta$ decay of the proton:

$$
\bar{\nu}_{e}+p \rightarrow e^{+}+n
$$

has a reaction threshold of $\mathrm{E}_{\text {thres }}=1.80 \mathrm{MeV}$. At low energies we approximate the total cross section as:

$$
\sigma=\frac{\mathrm{G}_{\mathrm{F}}^{2} \mathrm{p}_{e} \mathrm{E}_{e}}{\pi}\left|\cos ^{2} \theta_{c}\right|^{2}\left[1+3\left(\frac{\mathrm{g}_{\mathrm{A}}}{\mathrm{g}_{\mathrm{V}}}\right)^{2}\right],
$$

which can be re-written with appropriate parameters as:

$$
\sigma\left(\mathrm{E}_{\nu}\right)=9.5 \times 10^{-44}\left(\mathrm{E}_{\nu[\mathrm{MeV}]}-1.29\right)^{2} \mathrm{~cm}^{2} .
$$

Integrating this cross section with the $\bar{\nu}_{e}$ spectrum gives an event rate of about 79 neutrinos in Borexino from a typical supernova burst.

\section{$2.3{ }^{12}$ C reactions}

The $A=12$ isobar level scheme is shown in Fig. 1. Superallowed transitions leading from the ${ }^{12} \mathrm{C}\left(0^{+}, 0\right)$ ground state to the triad of $\left(1^{+}, 1\right)$ states: ${ }^{12} \mathrm{~N}_{\text {g.s. }}$, ${ }^{12} \mathrm{~B}_{\text {g.s. }}$ and ${ }^{12} \mathrm{C}^{*}(15.11 \mathrm{MeV})$ give rise to the following three neutrino reactions:

1) charged-current capture of $\bar{\nu}_{e}$ :

$$
\begin{array}{ll}
\bar{\nu}_{e}+{ }^{12} \mathrm{C} \rightarrow{ }^{12} \mathrm{~B}+e^{+} & \mathrm{Q}=14.39 \mathrm{MeV} \\
{ }^{12} \mathrm{~B} \rightarrow{ }^{12} \mathrm{C}+e^{-}+\bar{\nu}_{e} & \tau_{1 / 2}=20.20 \mathrm{~ms}
\end{array}
$$

2) charged-current capture of $\nu_{e}$ : 


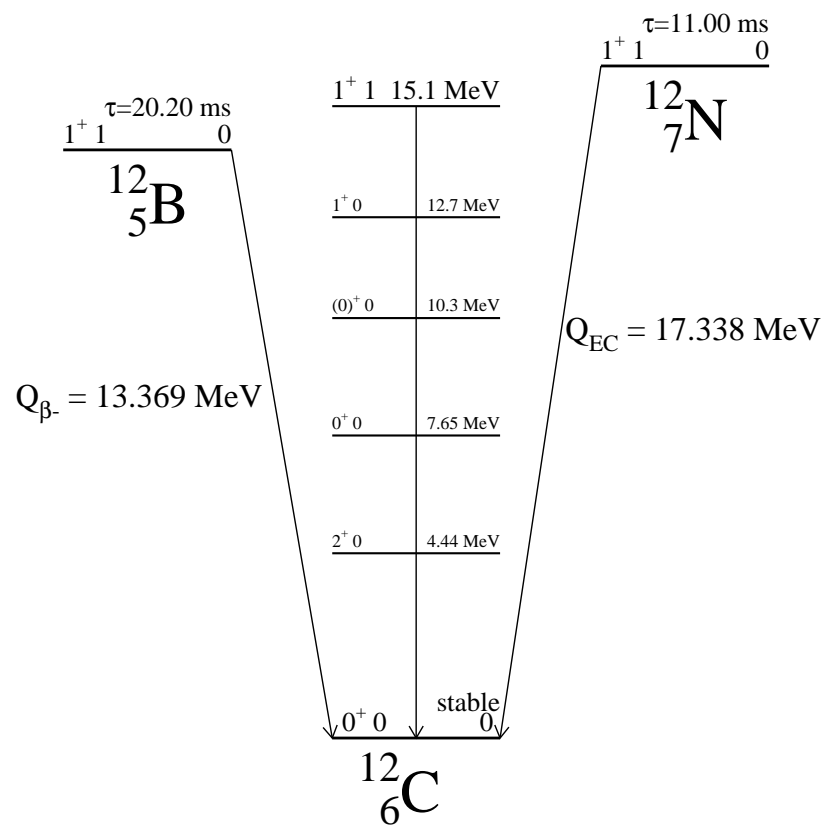

Fig. 1. Level diagram for the ${ }^{12} \mathrm{C},{ }^{12} \mathrm{~N},{ }^{12} \mathrm{~B}$ triad.

$$
\begin{array}{ll}
\nu_{e}+{ }^{12} \mathrm{C} \rightarrow{ }^{12} \mathrm{~N}+e^{-} & \mathrm{Q}=17.34 \mathrm{MeV} \\
{ }^{12} \mathrm{~N} \rightarrow{ }^{12} \mathrm{C}+e^{+}+\nu_{e} & \tau_{1 / 2}=11.00 \mathrm{~ms}
\end{array}
$$

3) neutral-current inelastic scattering of $\nu$ or $\bar{\nu}$ :

$$
\begin{array}{cc}
\nu+{ }^{12} \mathrm{C} \rightarrow{ }^{12} \mathrm{C}^{*}+\nu^{\prime} & \mathrm{Q}=15.11 \mathrm{MeV} \\
{ }^{12} \mathrm{C}^{*} \rightarrow{ }^{12} \mathrm{C}+\gamma & (15.11 \mathrm{MeV}) .
\end{array}
$$

All of the reactions on carbon can be tagged in Borexino. The charged-current events have the delayed coincidence of a $\beta$ decay following the interaction. The neutral-current events have a monoenergetic $\gamma$ ray at $15.1 \mathrm{MeV}$.

In the low-energy limit $(\mathrm{E} \ll 250 \mathrm{MeV})$ only superallowed and allowed transitions are significant; the charged-current cross section is given by:

$$
\sigma=\frac{\mathrm{G}_{\mathrm{F}}^{2}}{\pi} \cos ^{2} \theta_{c} \sum_{\mathrm{i}}\left|\mathrm{M}_{\mathrm{i}}\right|^{2} \mathrm{p}_{e} \mathrm{E}_{e} \mathrm{~F}\left(\mathrm{Z}, \mathrm{E}_{e}\right)
$$

where $\left|\mathrm{M}_{\mathrm{i}}\right|^{2}$ are the nuclear matrix elements squared and $\mathrm{F}\left(\mathrm{Z}, \mathrm{E}_{e}\right)$ is the Fermi function, accounting for Coulomb corrections in $\beta$ decays. For the neutralcurrent reaction only the isovector axial current contributes to the interaction; 
Table 2

Neutrino-carbon cross sections as measured and calculated by different authors, in units of $10^{-42} \mathrm{~cm}^{2}$. These are averaged over the experimental neutrino spectrum originating from muon decay at rest.

\begin{tabular}{lcc}
\hline & ${ }^{12} \mathrm{C}\left(\nu_{e}, e^{-}\right)^{12} \mathrm{~N}_{\text {g.s. }}$ & ${ }^{12} \mathrm{C}\left(\nu, \nu^{\prime}\right)^{12} \mathrm{C}^{*}\left[\nu=\nu_{e}+\bar{\nu}_{\mu}\right]$ \\
\hline EPT [10,13] & 9.2 & 9.9 \\
Shell model [14] & 9.1 & 9.8 \\
RPA [18] & 9.3 & 10.5 \\
KARMEN [19] & $8.9 \pm 0.6 \pm 0.75$ & $11 \pm 1.0 \pm 0.9$ \\
LAMPF [20] & $10.5 \pm 1.0 \pm 1.0$ & - \\
LSND [21] & $9.1 \pm 0.4 \pm 0.9$ & - \\
\hline
\end{tabular}

the cross section is given by:

$$
\sigma=\frac{\mathrm{G}_{\mathrm{F}}^{2}}{\pi} \sum_{\mathrm{i}}\left|\mathrm{M}_{\mathrm{i}}\right|^{2}\left(\mathrm{E}_{\nu}-\mathrm{E}_{\mathrm{i}}\right)^{2} .
$$

The cross sections for the neutrino-carbon reactions have been investigated theoretically and experimentally over the past 20 years and are now well established. The nuclear matrix element is the Gamow-Teller matrix element, determined at $q^{2}=0$ by the $\beta$ decay rates, in the Elementary Particle Treatment $[10,13]$. The resulting cross sections are confirmed also by the other two theoretical approaches: the Shell Model [14-17] and the Random Phase Approximation [18].

Measurements of cross sections for ${ }^{12} \mathrm{C}\left(\nu_{e}, e^{-}\right){ }^{12} \mathrm{~N}$ and ${ }^{12} \mathrm{C}\left(\nu, \nu^{\prime}\right){ }^{12} \mathrm{C}^{*}$ have also been performed at KARMEN [19], at LAMPF [20], and by LSND [21]. Table 2 compares the theoretical predictions to the measured data. The agreement is good and we based our rate calculations on a combined theoretical and experimental average of $\langle\sigma\rangle_{\exp }=9.2 \times 10^{-42} \mathrm{~cm}^{2}$. The cross section measurements were averaged over the neutrino energies relevant to the experiments, namely neutrinos from muon decay at rest. It is straightforward to scale these measured values to give averaged cross sections for supernova neutrinos.

Since ${ }^{12} \mathrm{~N}$ and ${ }^{12} \mathrm{~B}$ are "mirror nuclei", the matrix elements and energy-independent terms in the cross section are essentially identical. Only the Coulomb correction differs when calculating the $\bar{\nu}_{e}$ capture rates.

The neutral-current cross section can also be extracted from the experimental ${ }^{12} \mathrm{C}\left(\nu, \nu^{\prime}\right){ }^{12} \mathrm{C}^{*}$ cross section data. Note that the quoted values for $\langle\sigma\rangle$ from Table 2 are averaged over the experimental neutrino flux including both $\nu_{e}$ and $\bar{\nu}_{\mu}$ from muon decay. Using an averaged value $\langle\sigma\rangle_{\exp }=10 \times 10^{-42} \mathrm{~cm}^{2}$ and assuming the contribution of $\nu_{e}$ and $\bar{\nu}_{\mu}$ to the nuclear inelastic scattering 
Table 3

Supernova neutrino events in Borexino from a supernova at $10 \mathrm{kpc}$, with $\varepsilon_{B}=$ $3 \times 10^{53}$ ergs binding energy release.

\begin{tabular}{cccc}
\hline reaction channel & $\left\langle\mathrm{E}_{\nu}\right\rangle[\mathrm{MeV}]$ & $\langle\sigma\rangle\left[\mathrm{cm}^{2}\right]$ & $\mathrm{N}_{\text {events }}$ \\
\hline$\nu_{e}-e$ & 11 & $1.02 \times 10^{-43}$ & 2.37 \\
$\bar{\nu}_{e}-e$ & 16 & $6.03 \times 10^{-44}$ & 0.97 \\
$\nu_{x}-e$ & 25 & $3.96 \times 10^{-44}$ & 0.81 \\
$\bar{\nu}_{x}-e$ & 25 & $3.25 \times 10^{-44}$ & 0.67 \\
\hline total $\nu-e$ & & & 4.82 \\
\hline $\bar{\nu}_{e}+p \rightarrow e^{+}+n$ & 16 & $2.70 \times 10^{-41}$ & 79 \\
\hline${ }^{12} \mathrm{C}\left(\nu_{e}, e^{-}\right)^{12} \mathrm{~N}$ & & & \\
${ }^{12} \mathrm{C}\left(\bar{\nu}_{e}, e^{+}\right)^{12} \mathrm{~B}$ & 11 & $1.85 \times 10^{-43}$ & 0.65 \\
& 16 & $1.87 \times 10^{-42}$ & 3.8 \\
\hline total ${ }^{12} \mathrm{C}\left(\nu, \nu^{\prime}\right)^{12} \mathrm{C}^{*}$ & & & \\
\hline$\nu_{x}+{ }^{12} \mathrm{C}$ & & & \\
\hline $\bar{\nu}_{e}+{ }^{12} \mathrm{C}$ & & & \\
\hline
\end{tabular}

to be the same, we scaled this data for supernova neutrino fluxes and energies.

Table 3 summarizes our results; we estimate 23 neutral-current events, 4 events due to $\bar{\nu}_{e}$ capture on ${ }^{12} \mathrm{C}$ and less than one event due to $\nu_{e}$ capture, from a typical Galactic supernova at $10 \mathrm{kpc}$.

\section{Neutral Current Detection in Borexino}

The neutrino burst from a supernova rises steeply and decays exponentially in time: $\mathrm{L}_{\nu} \sim \mathrm{e}^{-t / \tau_{\nu}}$, with $\tau_{\nu} \approx 3 \mathrm{~s}[22]$. In a low-background solar neutrino detector, a burst of 100 events in a time window of 10 seconds is easily identified. The ability to separate the neutral-current events in a liquid scintillator detector from the $\bar{\nu}_{e}-p$ reactions determines whether interesting neutrino physics can be explored. 
The inverse $\beta$ decay of the proton produces a neutron. In Borexino, this neutron thermalizes and walks in the detector until it is captured by hydrogen: $n+p \rightarrow d+\gamma$, with a mean capture time $\tau=250 \mu \mathrm{s}$ and $\mathrm{E}_{\gamma}=2.2 \mathrm{MeV}$. The large homogeneous detection volume in Borexino ensures efficient neutron capture and efficient detection of the $2.2 \mathrm{MeV} \gamma$. These events can be tagged by the delayed coincidence between the initial $e^{+}$from the $p\left(\bar{\nu}_{e}, e^{+}\right) n$ reaction and the neutron capture $\gamma$ ray.

On the other hand, if a detector lacks the low-energy threshold of Borexino or a detector is not able to contain the neutron produced by the $\bar{\nu}_{e}-p$ reaction, it will not be able to exploit the delayed coincidence signature to identify these events. Consequently, the $\bar{\nu}_{e}-p$ events appear as single positrons. The challenge in such a detector is then to distinguish the $15.1 \mathrm{MeV} \gamma$ of ${ }^{12} \mathrm{C}$ neutral-current excitation from the continuum spectrum produced by these positrons.

Efficient detection and resolution of the $15.1 \mathrm{MeV} \gamma$ will also be possible in Borexino. Fig. 2 depicts an example of the singles spectrum from all supernova neutrino events that would occur in Borexino within a time window of 10 seconds. Even if the $\bar{\nu}_{e}-p$ positrons are not tagged by the delayed neutron capture $\gamma$ ray, the $15.1 \mathrm{MeV}$ peak is well resolved on top of the positron spectrum. The energy resolution in Borexino, simulated here with the design light collection statistics of 400 photoelectrons $/ \mathrm{MeV}$, allows the neutral-current events to be identified. The large, homogeneous volume of liquid scintillator effectively contains the total energy of this $\gamma$ ray.

\section{Consequences of Non-Standard Neutrino Physics}

\subsection{Neutrino Mass from Time of Flight}

The present limits on neutrino mass, obtained by laboratory experiments, are high for $\nu_{\mu}$ and $\nu_{\tau}: \mathrm{m}_{\nu_{e}}<3.9 \mathrm{eV}, \mathrm{m}_{\nu_{\mu}}<170 \mathrm{keV}$ and $\mathrm{m}_{\nu_{\tau}}<18.2 \mathrm{MeV}[23]$. By studying the arrival time of neutrinos of different flavors from a supernova, mass limits on $\nu_{\mu}$ and $\nu_{\tau}$ down to the tens of $\mathrm{eV}$ level can be explored.

Consider a supernova neutrino flux composed of two species, one massive and the other essentially massless. The massive neutrinos will reach Earth with a time delay:

$$
\Delta \mathrm{t}=\frac{\mathrm{D}}{2 \mathrm{c}}\left(\frac{\mathrm{m}_{\nu}}{\mathrm{E}_{\nu}}\right)^{2}
$$



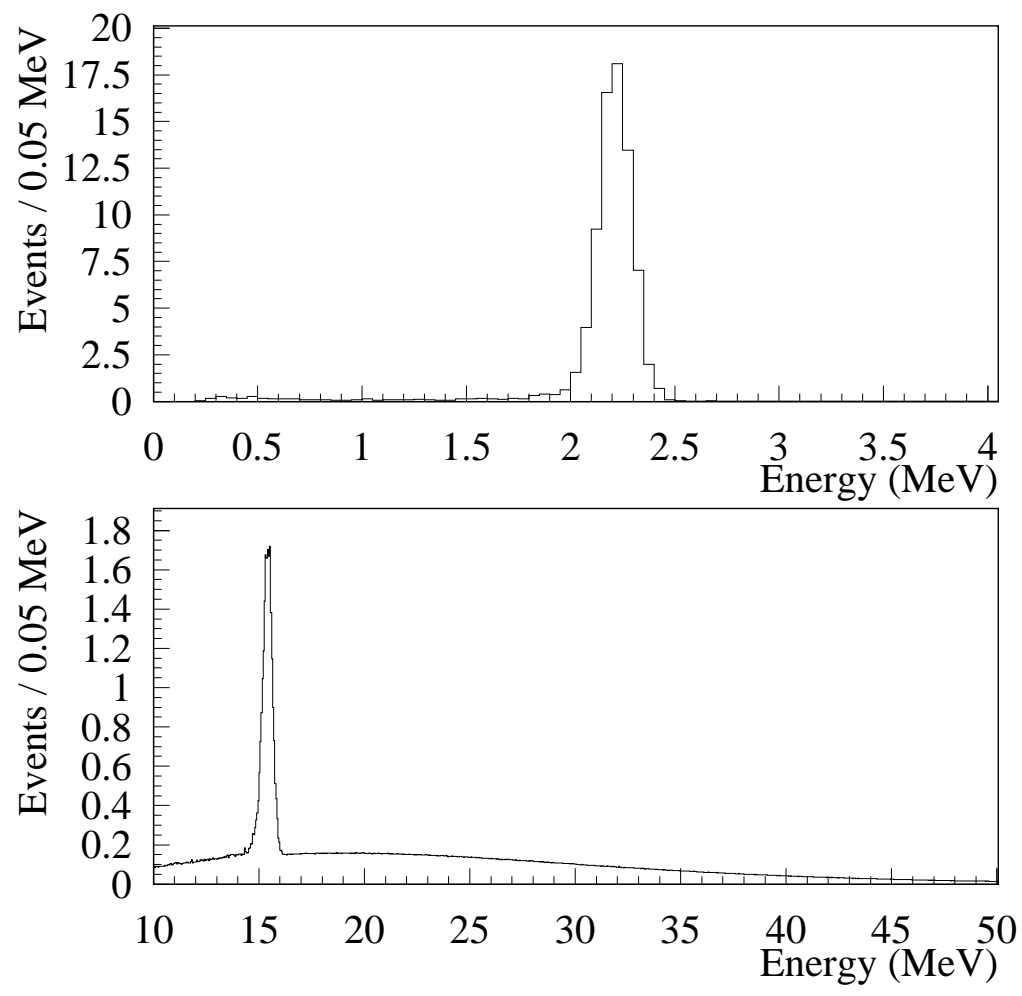

Fig. 2. Simulated singles spectrum from supernova neutrinos in Borexino, in two different energy windows. In the lower energy spectrum there will be a peak at $2.2 \mathrm{MeV}$, due to the capture of neutrons produced in the charged current $p\left(\bar{\nu}_{e}, e^{+}\right) n$ reaction. In the higher energy end of the spectrum $(\mathrm{E}>10 \mathrm{MeV})$, the $15.1 \mathrm{MeV} \gamma$ rays from neutral-current excitation of carbon are well resolved from the continuum of $e^{+}$events from $p\left(\bar{\nu}_{e}, e^{+}\right) n$.

with respect to the massless species, where $\mathrm{D}$ is the distance to the supernova. Measuring this time delay requires being able to distinguish the massive species from the massless neutrino interactions. Ideally, knowledge of the emission time distribution is also required as is a precise measurement of $\mathrm{E}_{\nu}$.

In Borexino, the neutral-current excitation is dominated by $\nu_{\mu}, \bar{\nu}_{\mu}, \nu_{\tau}$ and $\bar{\nu}_{\tau}$, due to their higher average energy; $91 \%$ of the neutral-current events come from the "heavy flavor" neutrinos. Their relative contribution to the neutralcurrent event rate is illustrated in Fig. 3. The $\bar{\nu}_{e}-p$ charged-current events provide the time stamp for the "massless" species (possibly even the $\bar{\nu}_{e}-p$ events from SuperKamiokande). Thus, in Borexino, determining the time delay between the neutral-current and charged-current events provides a handle on the mass of $\nu_{\mu}$ and/or $\nu_{\tau}$. 


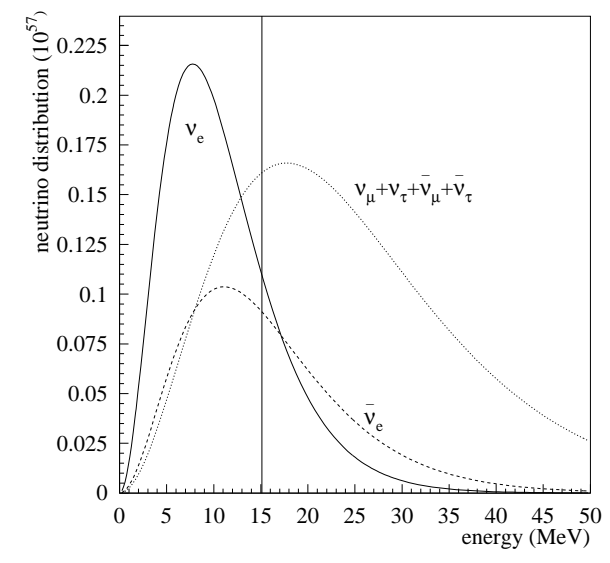

(a) $\mathrm{N}\left(\mathrm{E}_{\nu}\right)$

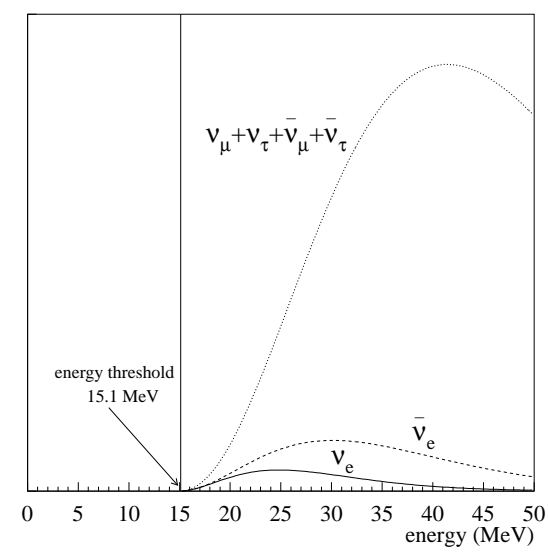

(b) $\mathrm{N}\left(\mathrm{E}_{\nu}\right) \sigma\left(\mathrm{E}_{\nu}\right)$

Fig. 3. Contribution of the different neutrino flavors to the neutral-current reaction ${ }^{12} \mathrm{C}\left(\nu, \nu^{\prime}\right){ }^{12} \mathrm{C}^{*}(15.11 \mathrm{MeV})$ : (a) supernova neutrino energy spectra and (b) their product with the cross section above threshold. The solid line is the $\nu_{e}$ distribution; the dashed line is the $\bar{\nu}_{e}$ profile; and the dotted line is the summed contribution of the other flavors $\left(\nu_{\mu}, \nu_{\tau}, \bar{\nu}_{\mu}\right.$ and $\left.\bar{\nu}_{\tau}\right)$. The reaction threshold is represented by the vertical line.

Note that no energy information, $\mathrm{E}_{\nu}$, is available from these neutral-current events. Additionally, no distinction between $\nu_{\mu}$ and $\nu_{\tau}$ is possible. Nevertheless, the overall rate of these events constrains the average energy of these neutrinos, provided that the distance to the supernova and the overall luminosity can be determined by other means (and given the assumptions outlined earlier, such as equipartition of luminosity and a thermal distribution of energies).

Beacom and Vogel have shown $[24,25]$ that model-specific details relating to the emission time profile of neutrinos from a supernova do not have a pronounced effect on the arrival time distribution. They demonstrate that the overwhelming consideration in analyzing the time delay for massive neutrinos is the time constant of the exponential decay of the neutrino luminosity. They show, in addition, that averaged quantities such as:

$$
\Delta t=\langle t\rangle_{N C}-\langle t\rangle_{C C}
$$

can be used as sensitive probes for extraction of the neutrino mass from supernova neutrino data.

We considered a model for a supernova neutrino burst that rises linearly, reaching maximum in the first $20 \mathrm{~ms}$. This is followed by an exponential decay with $\tau=3 \mathrm{~s}[22]$. Fig. 4 shows the expected time distribution of the ${ }^{12} \mathrm{C}\left(\nu, \nu^{\prime}\right){ }^{12} \mathrm{C}^{*}$ events in Borexino, for two scenarios. In both cases, $\nu_{e}$ is assumed massless and: 

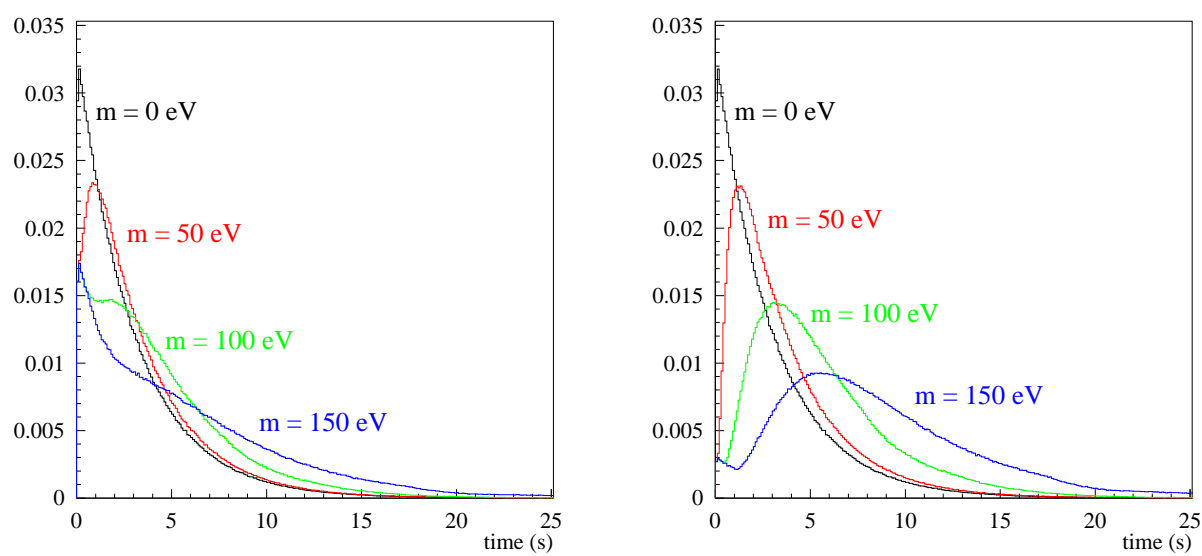

(a) only $\mathrm{m}_{\nu_{\tau}} \neq 0$

(b) $\mathrm{m}_{\nu_{\mu}} \neq 0, \mathrm{~m}_{\nu_{\tau}} \neq 0$

Fig. 4. Time distribution for the ${ }^{12} \mathrm{C}\left(\nu, \nu^{\prime}\right){ }^{12} \mathrm{C}^{*}$ events, for two cases: (a) $46 \%$ of the events are from massive neutrinos $\left(\nu_{\tau}+\bar{\nu}_{\tau}\right)$; (b) $91 \%$ of the events are massive $\left(\nu_{\mu}+\bar{\nu}_{\mu}\right.$ and $\left.\nu_{\tau}+\bar{\nu}_{\tau}\right)$.

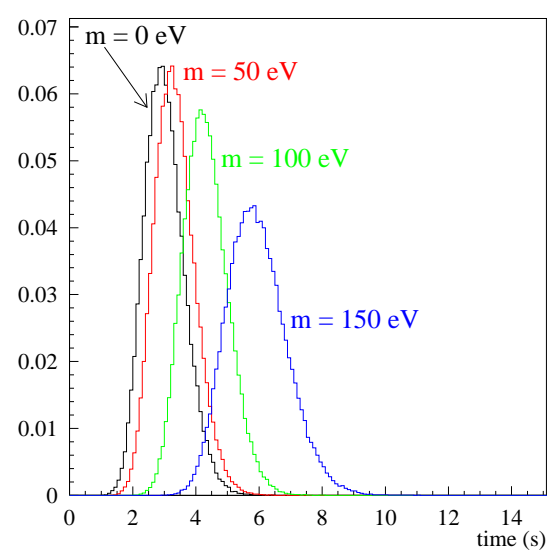

(a) only $\mathrm{m}_{\nu_{\tau}} \neq 0$

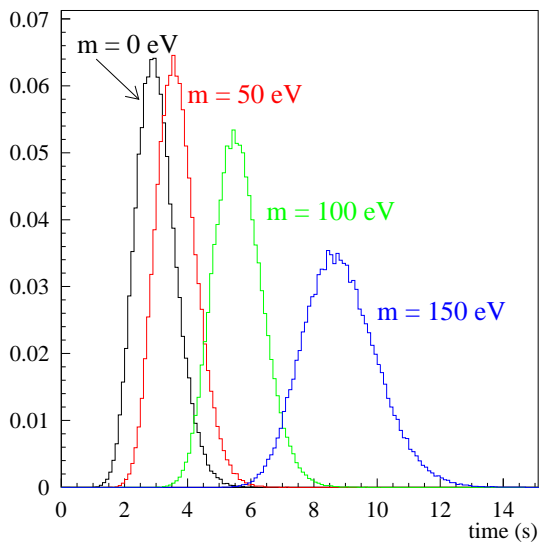

(b) $\mathrm{m}_{\nu_{\mu}} \neq 0, \mathrm{~m}_{\nu_{\tau}} \neq 0$

Fig. 5. Monte Carlo distribution of the average time of the ${ }^{12} \mathrm{C}\left(\nu, \nu^{\prime}\right){ }^{12} \mathrm{C}^{*}$ events, for a sample of $10^{5}$ supernovae at $10 \mathrm{kpc}$ (in this model, $t=3 \mathrm{~s}$ is the average arrival time of the massless neutrinos).

(a) $\nu_{\mu}$ is massless and $\nu_{\tau}$ is massive $\Rightarrow 46 \%$ of the ${ }^{12} \mathrm{C}\left(\nu, \nu^{\prime}\right)^{12} \mathrm{C}^{*}$ events are delayed;

(b) $\nu_{\mu}$ and $\nu_{\tau}$ are both massive $\Rightarrow 91 \%$ of the ${ }^{12} \mathrm{C}\left(\nu, \nu^{\prime}\right){ }^{12} \mathrm{C}^{*}$ events are delayed.

Fig. 5 shows the distribution of average time of arrival of the ${ }^{12} \mathrm{C}\left(\nu, \nu^{\prime}\right){ }^{12} \mathrm{C}^{*}$ 


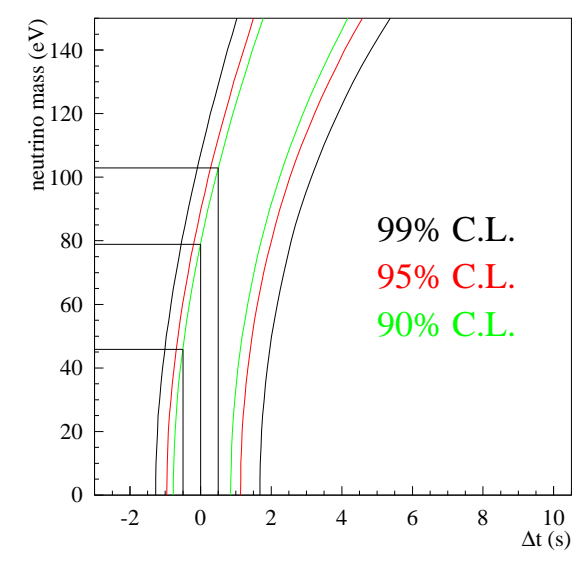

(a) only $\mathrm{m}_{\nu_{\tau}} \neq 0$

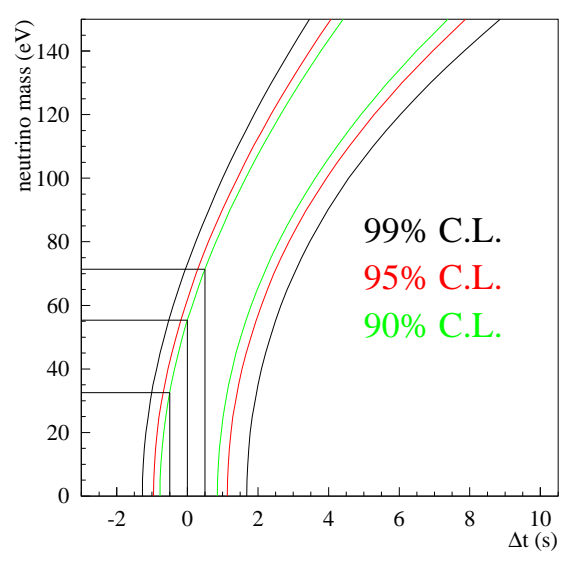

(b) $\mathrm{m}_{\nu_{\mu}} \neq 0, \mathrm{~m}_{\nu_{\tau}} \neq 0$

Fig. 6. Contour probability plots relating the average delay of ${ }^{12} \mathrm{C}\left(\nu, \nu^{\prime}\right){ }^{12} \mathrm{C}^{*}$ events to the neutrino mass, in the two scenarios. In both cases, the vertical lines correspond to a measured delay between charged- and neutral-current reactions equal to -0.5 , 0 and $+0.5 \mathrm{~s}$. Their intersections with the contour plots set limits on the neutrino mass with $90 \%, 95 \%$ and $99 \%$ C.L. For instance, if no delay is measured between the $p\left(\bar{\nu}_{e}, e^{+}\right) n$ charged-current events and the ${ }^{12} \mathrm{C}\left(\nu, \nu^{\prime}\right){ }^{12} \mathrm{C}^{*}$ neutral-current events, we can state with $90 \%$ C.L. that $m_{\nu_{\tau}}<79 \mathrm{eV}$ in scenario (a) or $m_{\nu_{\mu \tau}}<55 \mathrm{eV}$ in scenario (b).

events, for different values of the heavy neutrino mass. It is based on the results of a Monte Carlo simulation of $10^{5}$ supernovae (distance $10 \mathrm{kpc}$ ) producing ${ }^{12} \mathrm{C}$ neutral-current events in Borexino. The arrival times for the, on average, 23 events were drawn from the distributions shown in Fig. 4. Time zero is the theoretical instant of the earliest possible arrival (possibly determined by the earliest detection of $\bar{\nu}_{e}$ at SuperKamiokande, corrected for flight time). To obtain $\Delta t$, we subtract the average arrival time of the light neutrinos, that is $\langle t\rangle_{C C}=3 \mathrm{~s}$.

Fig. 6 shows the probability contour plots for neutrino mass as a function of average arrival time delay. Different probability contours are included in the figure. The interpretation of these plots, in terms of neutrino mass limits, goes as follows. Consider scenario (a), where $\nu_{\tau}$ is massive (46\% of the neutralcurrent events are delayed). From the data set, the average arrival time of the charged-current events is subtracted from the average arrival time of the neutral-current events, giving a value for $\Delta t$. Given a measured delay (or lack thereof) allows one to set a mass limit, such that:

$$
\begin{aligned}
& \text { if } \Delta t=-0.5 \mathrm{~s} \text {, then } m_{\nu_{\tau}}<46 \mathrm{eV}(90 \% C L) \\
& \text { if } \Delta t=0 \mathrm{~s} \text {, then } m_{\nu_{\tau}}<79 \mathrm{eV}(90 \% C L)
\end{aligned}
$$




$$
\text { if } \Delta t=+0.5 \mathrm{~s} \text {, then } m_{\nu_{\tau}}<103 \mathrm{eV}(90 \% C L)
$$

Similarly, for scenario (b) in which both $\nu_{\tau}$ and $\nu_{\mu}$ are massive, with $m_{\nu_{\mu}} \simeq m_{\nu_{\tau}}$ (as might arise for small $\Delta m_{23}$ ) mass limits are extracted such that:

$$
\begin{aligned}
& \text { if } \Delta t=-0.5 \mathrm{~s}, \text { then } m_{\nu_{\mu \tau}}<33 \mathrm{eV}(90 \% C L) \\
& \text { if } \Delta t=0 \mathrm{~s}, \text { then } m_{\nu_{\mu \tau}}<55 \mathrm{eV}(90 \% C L) \\
& \text { if } \Delta t=+0.5 \mathrm{~s} \text {, then } m_{\nu_{\tau}}<71 \mathrm{eV}(90 \% C L)
\end{aligned}
$$

Conversely, a measured average arrival time delay of $\Delta t=+1.0 \mathrm{~s}$ allows one to exclude a massless $\nu_{\tau}$ at greater than $90 \%$ confidence level.

These limits are only slightly worse than what might be achievable in SuperKamiokande (a sensitivity of $50 \mathrm{eV}$ ) [24], and in SNO (a sensitivity of $30 \mathrm{eV}$ ) [25]. It is remarkable that the limits in Borexino are comparable, given the much higher statistics expected in these much larger detectors. Since $\Delta t \propto \mathrm{m}^{2}$, and the error on $\Delta t$ is proportional to the square root of the number of detected events, the error on the neutrino mass will, in the end, be proportional only to the fourth root of the number of detected events. This aspect combined with the higher "heavy-flavor fraction" in the neutral-current events in Borexino allow $\nu_{\tau}$ mass limits in the cosmologically-significant range to be reached.

These results, while being independent of many of the details in the supernova neutrino emission models, are nevertheless based on the assumptions made here on the supernova neutrino energy spectrum and intensity. In these calculations, the distance to the supernova was assumed to be precisely determined.

\subsection{Neutrino Oscillations}

Neutrino oscillations can be probed by comparing the supernova neutrino event rates for different reactions. The extent of limits on $\Delta \mathrm{m}^{2}$ depend on the $\mathrm{L} / \mathrm{E}$ ratio which, for distances of kiloparsecs, is many orders of magnitude lower than present regions explored (e.g. solar neutrino vacuum oscillations).

We will consider, as an example, the implications of vacuum oscillations in the solar neutrino sector on the detection of supernova neutrinos in Borexino. Since the actual phase of the oscillation can be any value (varies rapidly over these distance scales) we take an average value of: $\left\langle\sin ^{2}\left(\pi \mathrm{L} / \lambda_{\text {osc }}\right)\right\rangle_{\mathrm{L}}=0.5$.

The main consideration is that higher energy $\nu_{\mu}$ could oscillate into $\nu_{e}$, resulting in an increased event rate since the expected $\nu_{e}$ energies are just at or below the charged-current reaction threshold. The cross section for ${ }^{12} \mathrm{C}\left(\nu_{e}, e^{-}\right)^{12} \mathrm{~N}$ in- 


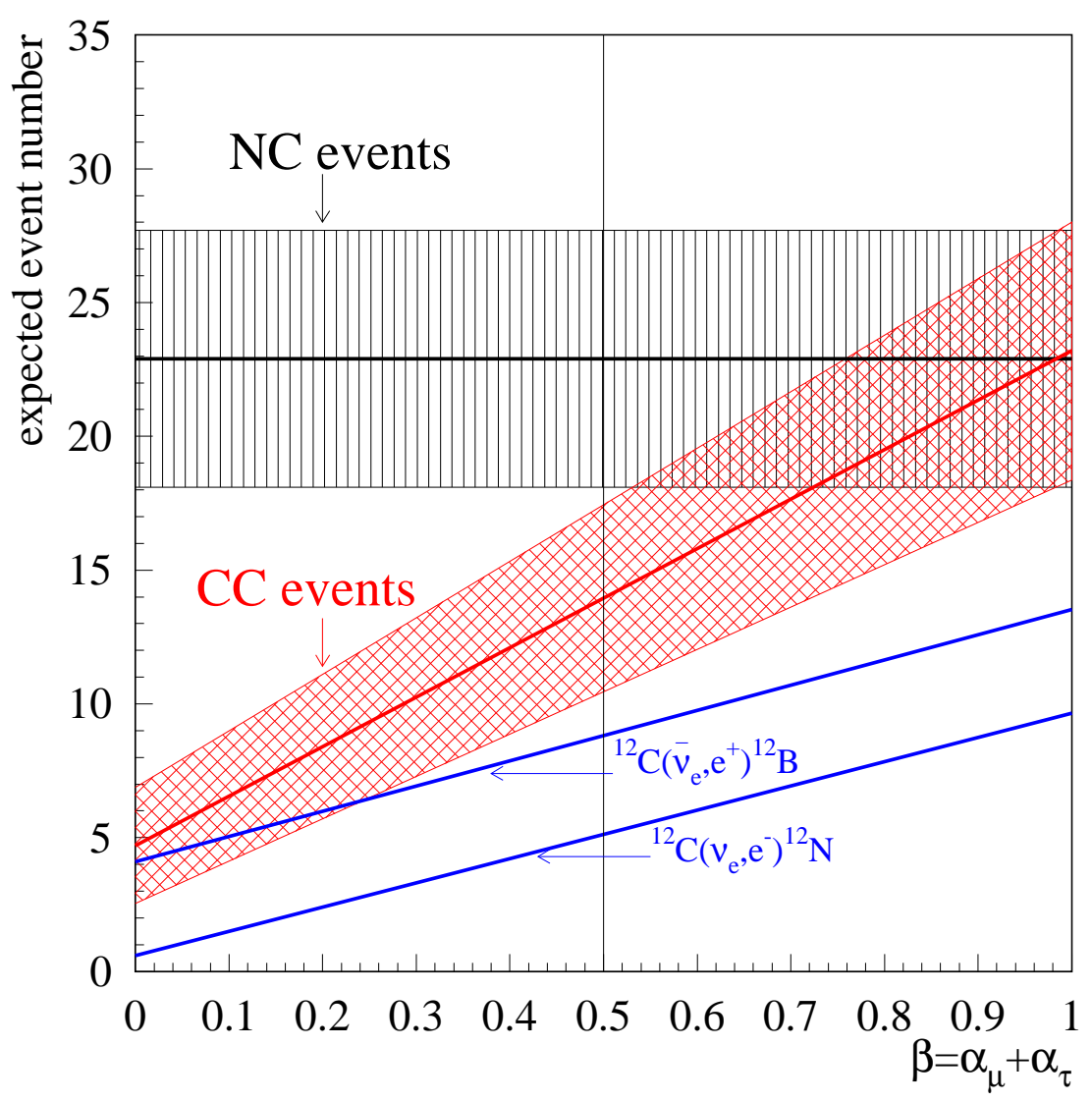

Fig. 7. Number of $\mathrm{NC}$ and $\mathrm{CC}$ events on ${ }^{12} \mathrm{C}$, as a function of an overall conversion probability $\beta=\alpha_{\mu}+\alpha_{\tau}$, where $\alpha_{\mu}=\mathrm{P}\left(\nu_{e} \leftrightarrow \nu_{\mu}\right)$ and $\alpha_{\tau}=\mathrm{P}\left(\nu_{e} \leftrightarrow \nu_{\tau}\right)$. The error bands refer to the case of a supernova at $10 \mathrm{kpc}$ with $\varepsilon_{B}=3 \times 10^{53} \mathrm{ergs}$.

creases by a factor of 35 if we average it over a $\nu_{e}$ distribution with $T=8 \mathrm{MeV}$, rather than $3.5 \mathrm{MeV}$. The gain in cross section for ${ }^{12} \mathrm{C}\left(\bar{\nu}_{e}, e^{+}\right)^{12} \mathrm{~B}$ is a factor of 5 . The large increase in the $\nu_{e}$ induced reaction rate is a pseudo-appearance signature for oscillations.

We assign the probability $\alpha_{\mu}$ to $\nu_{e} \leftrightarrow \nu_{\mu}$ conversion and $\alpha_{\tau}$ to be the $\nu_{e} \leftrightarrow \nu_{\tau}$ conversion probability, and define $\beta=\alpha_{\mu}+\alpha_{\tau}$. Thus, depending on the value of $\beta$, we have the combinations of event numbers reported in and displayed in Fig. 7; the number of charged-current and neutral-current events, as a function of the parameter $\beta$ are shown with a $1 \sigma$ error band corresponding to the statistics for a supernova at $10 \mathrm{kpc}$. The constant neutral-current rate fixes the flavor-independent luminosity. Though the statistics are low, the effect on the charged-current rates are significant. For instance, in the case of $\beta=0.5$, the number of ${ }^{12} \mathrm{C}\left(\nu_{e}, e^{-}\right)^{12} \mathrm{~N}$ events increases from 0.65 to 5 , while the number 
of ${ }^{12} \mathrm{C}\left(\bar{\nu}_{e}, e^{+}\right)^{12} \mathrm{~B}$ doubles, from 4 to 8 .

\section{Conclusions}

The Borexino detector will be sensitive to neutrinos from a Type II supernova in our galaxy. A typical supernova at a distance of $10 \mathrm{kpc}$ will produce about 80 events from $\bar{\nu}_{e}$ capture on protons and about 30 events from reactions on ${ }^{12} \mathrm{C}$, mostly from $\nu_{\mu}$ and $\nu_{\tau}$ neutral-current events. As a large homogeneous volume liquid scintillator, identification of the neutral-current events via detection of the monoenergetic $15.1 \mathrm{MeV} \gamma$ enables Borexino to explore non-standard neutrino physics. Neutrino masses in the tens of $\mathrm{eV}$ range can be explored for $\nu_{\mu}$ and $\nu_{\tau}$ by measuring the delayed arrival of the neutral-current signal which is predominantly due to the interactions of $\nu_{\mu}$ and $\nu_{\tau}$. The charged-current and neutral-current reactions on ${ }^{12} \mathrm{C}$ also offer an important tool for probing neutrino oscillations. The distinctive charged-current reactions feature delayed $\beta$ decays, correlated in time and space with the neutrino interactions, allowing both $\nu_{e}$ and $\bar{\nu}_{e}$ to be easily identified in a flavor-oscillation analysis.

At the time of submission of this manuscript, the authors learned of the calculation by Beacom et al. [26] that recoil protons from $\nu-p$ scattering might provide an abundant, detectable signal in Borexino. The general idea is that though weak neutral-current cross sections are lower, the neutrinos (and antineutrinos) from supernovae are abundant in all three flavors, making the total flux more than six times greater than the flux from just $\bar{\nu}_{e}$. Furthermore, the $\nu_{\mu}$ and $\nu_{\tau}$ flavors are more energetic, increasing the total event rate in this channel such that it even exceeds the $\bar{\nu}_{e}-p$ charged-current event rate. This provides Borexino with several hundred supernova neutrino interactions, in the event of a galactic supernova. The challenge is detection of the low-energy proton recoils. As Borexino is designed for a low energy threshold and since Borexino will exploit pulse-shape discrimination in the liquid scintillator, it is likely that the proton recoils will be cleanly detected. What this enables in Borexino is sensitivity to the energy in the neutral-current signal and much greater statistics.

\section{Acknowledgments}

The authors thank P. Vogel for early discussions and J.F. Beacom for interesting and valuable discussions of the arrival time profile for massive neutrinos from a supernova. This work was supported in part by the National Science Foundation. 


\section{References}

[1] R. Bionta et al., Phys. Rev. Lett. 58 (1987) 1494.

[2] K. Hirata et al., Phys. Rev. D 38 (1988) 448; K. Hirata et al., Phys. Rev. Lett. 58 (1987) 1490.

[3] Y. Totsuka, Rep. Prog. Phys. 55 (1992) 377; T. Totani, K. Sato, H.E. Dalhed, J.R. Wilson, Astrophys. J. 496 (1998) 216.

[4] J. Boger et al., Nucl. Instr. \& Methods A 449 (2000) 172; A.B. Balantekin, F. Loreti, Phys. Rev. D 45 (1992) 1059.

[5] E.N. Alekseev et al., Phys. Part. Nucl. 29 (1998) 254.

[6] W. Fulgione for the LVD Collaboration, Nucl. Phys. B (Proc. Suppl.) 70 (1999) 469; M. Aglietta et al., Il Nuovo Cimento A 105 (1992) 1793.

[7] M. Ambrosio et al., Astropart. Phys. 8 (1998) 123.

[8] C. Arpesella et al., INFN Borexino Proposal, Vols. 1 and 2, eds. G. Bellini, R.S. Raghavan, et al. (U. Milano, 1992). J.B. Benziger, F.P. Calaprice et al., NSF Borexino Proposal, (Princeton, 1992). J.B. Benziger, F.P. Calaprice et al., A Proposal for Participation in the Borexino Solar Neutrino Experiment, (Princeton, 1996).

[9] A. Suzuki for the KamLAND Collaboration, Nucl. Phys. B (Proc. Suppl.) 77 (1999) 171.

[10] M. Fukugita, Y. Kohyama, K. Kubodera, Phys. Lett. B 212 (1988) 139.

[11] K. Langanke, P. Vogel, E. Kolbe, Phys. Rev. Lett. 76 (1996) 2629.

[12] D.N. Schramm, Comm. Nucl. Part. Phys. 17 (1987) 239.

[13] J. Bernabeu, P. Pascual, Nucl. Phys. A 324 (1979) 365; S.L. Mintz, M. Pourkaviani, Phys. Rev. C 40 (1989) 2458.

[14] J. Engel, E. Kolbe, K. Langanke, P. Vogel, Phys. Rev. C 54 (1996) 2740.

[15] Woosley et al., Astrophys. J. 356 (1990) 272.

[16] T.W. Donnelly and R.D. Peccei, Neutral Current Effects in Nuclei, Phys. Rep. 50 (1979) 1.

[17] T.W. Donnelly, Phys. Lett. B 43 (1973) 93; J.S. O'Connell, T.W. Donnelly, J.D. Walecka, Phys. Rev. C 6 (1972) 719.

[18] E. Kolbe, K. Langanke, S. Krewald, Phys. Rev. C 49 (1994) 1122; E. Kolbe, K. Langanke, F.K. Thielemann, P. Vogel Phys. Rev. C 52 (1995) 3437; E. Kolbe, K. Langanke, S. Krewald, F.K. Thielemann, Nucl. Phys. A 540 (1992) 599.

[19] B. Bodman et al., Phys. Lett. B 267 (1991) 321; KARMEN collaboration, Prog. Part. Nucl. Phys. 32 (1994) 351. 
[20] D.A. Krakauer et al., Phys. Rev. C 45 (1992) 2450; R.C. Allen et al., Phys. Rev. Lett. 64 (1990) 1871.

[21] C. Athanassopoulos et al., Phys. Rev. C 55 (1997) 2078.

[22] A. Burrows et al., Phys. Rev. D 45 (1992) 3361;

[23] Particle Data Group, Eur. Phys. J. C 3 (1998) 1.

[24] J.F. Beacom and P. Vogel, Phys. Rev. D 58 (1998) 053010.

[25] J.F. Beacom and P. Vogel, Phys. Rev. D 58 (1998) 093012.

[26] J.F. Beacom, private communication, September 21, 2000. 